\title{
Performance of Various Commercial Assays for the Detection of Toscana Virus Antibodies
}

\author{
Koray Ergünay, ${ }^{1}$ Nadine Litzba, ${ }^{2}$ Modou Moustapha Lo, ${ }^{3}$ Sibel Aydoğan, Mehmet B. Saygan, ${ }^{4}$ \\ Dürdal Us, Manfred Weidmann, ${ }^{3}$ and Matthias Niedrig ${ }^{2}$
}

\begin{abstract}
Introduction: Sandfly fever virus (SFV) serotypes sandfly fever Naples virus, sandfly fever Sicilian virus, and sandfly fever Cyprus virus cause febrile diseases, whereas Toscana virus (TOSV) is responsible for aseptic meningoencephalitis. Diagnosis and surveillance of TOSV depend heavily on virus serology, and various commercial assays utilizing various antigen sources and formats have been available. The aim of this study was to perform comparative evaluation of commercially available serological assays for anti-TOSV immunoglobulins.

Materials and Methods: A collection of 120 sera from healthy blood donors from an endemic region, previously identified to be reactive for antibodies against various SFV serotypes by indirect immunofluorescence test (IIFT), was reevaluated for IgG/IgM via IIFT, enzyme-linked immunosorbent assay, and an immunoblot assay manufactured by Euroimmun, Diesse, and Mikrogen, respectively. Virus neutralization test (VNT) was performed for 99 sera using standard TOSV, sandfly fever Sicilian virus, and sandfly fever Naples virus strains.

Results: A total of 89 samples $(74.2 \%)$ were reactive for TOSV IgG in at least one of the commercial assays, and 31 samples (31.3\%) were reactive in VNT for various SFV serotypes. Average percentage agreements among commercial assays and between VNT and the commercial assays were noted as $57.8 \%$ and $62.6 \%$, respectively. No significant correlation between assay results and VNT titers was observed. SFV IgM antibodies were detected in a total of eight samples $(6.7 \%)$ via IIFT, which were nonreactive in enzyme-linked immunosorbent assay and VNT.

Discussion: Commercial diagnostic immunoassays displayed slight to fair agreement for TOSV IgG as assessed via kappa and percentage agreement values. The results could only be confirmed via virus neutralization in a portion of the samples, and overall agreement between the commercial assays and VNT was slight. Commercial assays such as immunoblot can be used in addition to VNT for confirmation of TOSV exposure.
\end{abstract}

Key Words: Diagnosis—Sandfly fever virus—Serology—SFV—Toscana virus—TOSV.

\section{Introduction}

S ANDFLY FEVER VIRUSES (SFVs; family Bunyaviridae, genus Phlebovirus) are arthropod-borne viruses transmitted to humans by the bite of phlebotomine sandflies (Mertz 1997). Certain SFV serotypes, namely, sandfly fever Sicilian virus (SFSV), sandfly fever Naples virus (SFNV), and sandfly fever Cyprus virus (SFCV), are responsible for a self-limited febrile condition, whereas Toscana virus (TOSV) displays a distinct neurotropism and may cause aseptic meningoencephalitis in the infected individuals (Dionisio et al. 2003). In endemic regions, such as countries in the Mediterranean basin, exposure to SFVs is common as revealed by serosurveys, and TOSV infections account for a high proportion of human aseptic meningitis (Eitrem et al. 1991a, Nicoletti et al. 1996, Hemmersbach-Miller et al. 2004, Sanbonmatsu-Gamez et al. 2005).

In acute cases, methods for the diagnosis of TOSV infections include direct detection of the virus in patient samples either by culture or by nucleic acid amplification techniques and virus serology (Dionisio et al. 2003, Hemmersbach-Miller

\footnotetext{
${ }^{1}$ Department of Medical Microbiology, Virology Unit, Faculty of Medicine, Hacettepe University, Ankara, Turkey.

${ }^{2}$ Center for Biological Security (ZBS-1), Robert Koch Institute, Berlin, Germany.

${ }^{3}$ Institute of Virology, University Medical Center, Göttingen, Germany.

${ }^{4}$ Middle Anatolia Regional Blood Center, Turkish Red Crescent Society, Ankara, Turkey.
} 
et al. 2004, Sanbonmatsu-Gamez et al. 2005). Investigation of TOSV-specific immunoglobulins is also important for identifying virus exposure in healthy subjects from endemic countries or in regions where the sandfly vectors are present (Dionisio et al. 2003). The early phase of the SFV disease is characterized by a slow increase in specific IgMs, followed by IgG several days afterward in the serum (Echevarria et al. 2003). IgM can still be detected at least 1 year after the exposure, whereas IgG levels slowly increase, reaching a peak in the convalescent phase and persists for several years (Magurano and Nicoletti 1999). Various methods can be used for the detection of TOSV IgM and/or IgG antibodies, including hemagglutination inhibition, indirect immunofluorescence test (IIFT), enzyme-linked immunosorbent assay (ELISA), immunoblot (IB), and virus neutralization test (VNT) (Clarke and Cassals 1958, Eitrem et al. 1991b, Schwarz et al. 1995, 1996, Magurano and Nicoletti 1999). Some of these assays are commercially available and have been developed with partially purified antigens, infected cells, or recombinant viral proteins (Eitrem et al. 1991b, Schwarz et al. 1995, Schwartz et al. 1998, Ciufolini et al. 1999).

Exposure to a particular SFV serotype does not protect from infection with another serotype, and cross-reactions in serological tests can be frequently observed (Dionisio et al. 2003). Pre-existing antibodies against SFV serotypes other than TOSV (SFSV or SFNV) may also interfere with the TOSV serology results and can be interpreted as false-positive due to the cross-reactivity. This is more likely to occur when partially purified virus extracts or SFV-infected cells are employed as the source of antigens in the assay used for the diagnosis or surveillance (Schwarz et al. 1995, Magurano and Nicoletti 1999). During human immune response to TOSV infection, $N$ protein has been identified as the immunodominant antigen, which has later been used for developing diagnostic assays with recombinant proteins (Ciufolini et al. 1999, Cusi et al. 2001). The recombinant $N$ protein-based assays have been previously evaluated and have performed well, especially for acute TOSV infections (Valassina et al. 1998, Soldateschi et al. 1999). Nevertheless, cross-reactivity in phlebovirus serology affects the assays that employ TOSV N protein as well, especially in regions where different phleboviruses co-circulate, due to the presence of highly conserved regions in the viral nucleocapsid (Magurano and Nicoletti 1999, Di Bonito et al. 2002). To discriminate among related phleboviruses, it is recommended to complement recombinant $\mathrm{N}$-protein-based serodiagnosis with VNT (Calisher et al. 1999, Dionisio et al. 2003). However, phlebovirus neutralization has been shown to be mediated only partially by antibodies to the nucleocapsid, and immunity to viral envelope glycoproteins also plays a role (Cusi et al. 2001, Di Bonito et al. 2002). The immune response to viral glycoproteins has been shown to differ among patients, whereas all exposed persons develop antibodies against linear epitopes of $\mathrm{N}$ protein (Di Bonito et al. 2002). Interpretation of serological assay results in endemic regions may be more difficult, due to frequent recent infections and/or reinfections that boost immunity (Calisher et al. 1999, Dionisio et al. 2003).

The aim of this study was to evaluate the performances of all commercially available serological assays utilizing various antigen sources for detecting anti-TOSV antibodies, compared to virus neutralization in samples from a TOSV and SFV endemic region (Ergünay et al. 2010).

\section{Materials and Methods}

\section{Samples and commercial assays}

Serum samples reactive for SFV IgG by a commercial IIFT (SFV IgG mosaic I; Euroimmun) performed during a serosurveillance study in Central Anatolia, Turkey, were employed in the study (Ergünay et al. 2010). The samples originated from healthy blood donors and were obtained after informed consent. The IIFT allows simultaneous detection of antibodies against four viral serotypes (SFSV, SFNV, TOSV, and SFCV) and was performed as indicated by the manufacturer. A total of 120 samples were retested via this assay before inclusion in the study and reactive sera were divided into six panels according to the IIFT IgG reactivity against different viral serotypes: Panel 1: SFSV (n:18); Panel 2: SFNV (n:11); Panel 3: TOSV (n:46); Panel 4: SFCV (n:7); Panel 5: SFSV+SFCV (n:13); and Panel 6: SFNV+TOSV (n:25). All samples were further evaluated for SFV IgM by a commercial IIFT (SFV IgM Mosaic I; Euroimmun), for TOSV IgM/IgG by a commercial ELISA (TOSV IgG and IgM; Diesse) and by a commercial IB assay for TOSV IgG (recomLine Bunyavirus IgG/IgM; Mikrogen) according to the manufacturers' instructions. In addition to TOSV, antibodies to certain Hantavirus serotypes (Hantaan, Puumala, Dobrava, and Seoul) can also be simultaneously detected via the IB. In the IIFT, antibody levels were determined starting at the serum dilution of 1:100. The positive results were evaluated as equivocal $(1 / 100)$, positive $(1 / 320)$, or strong positive $(1 / 1000$ or higher) according to visual intensity of fluorescence compared to control sera. In the ELISA, qualitative evaluation of the results was performed by comparing the average optic density of the sample with the cutoff control. When the sample to cutoff ratio was $>1.2$, the sample was interpreted as positive and borderline if the ratio was between 1.2 and 0.8 . The IB results are interpreted according to the visual intensity of the cutoff control where intensities equal to the cutoff as positive $(+)$ and stronger than the cutoff as strong positive $(++,+++)$ as indicated by the manufacturer. The general features and performance characteristics of the commercial assays are provided in Table 1.

\section{Virus neutralization test}

VNT for SFVs were performed in 96-well microtiter plates with Vero cells (ATCC CCL81) and was adapted from the VNT used for Rift Valley fever virus described previously (Davies et al. 1988). Serial serum dilutions of $1 / 10$ to $1 / 320$ and virus suspensions of $10^{8} \mathrm{TCID}_{50}$ were prepared with standard strains (SFSV Oct-85 Sabin, SFNV Oct-85 Sabin and TOSV ISS.Phl.3). The viruses were then diluted to contain a final concentration of $100 \mathrm{TCID}_{50}$ per $50 \mu \mathrm{L}$. The diluted virus and serum dilutions were distributed into all wells excepted for the controls. After an hour of incubation at $37^{\circ} \mathrm{C}$, Vero cells $\left(\sim 2.10^{5}\right.$ in number per well) in $10 \%$ fetal calf serum were added to the serum-virus complex. Cells were evaluated after 72 and $96 \mathrm{~h}$ of incubation at $37^{\circ} \mathrm{C}$, and reactivity was determined by the lack of cytopathogenic effect above a serum dilution of $1 / 20$ at the 96 th hour.

\section{Statistical analysis}

Agreement among commercial assays by the Kappa, Fleiss' Kappa coefficients (Landis and Koch 1977, Ilstrup 1990), and 
Table 1. General Characteristics of the Commercial Assays Evaluated in the Study

\begin{tabular}{|c|c|c|c|}
\hline & IIFT & ELISA & $I B$ \\
\hline Manufacturer & Euroimmun Labordiagnostika AG & Diesse Diagnostica & Mikrogen Diagnostik \\
\hline Assay format & Immunofluorescent assay & Enzyme immunoassay & Strip immunoassay \\
\hline Antigen source & Cell culture grown viruses & Recombinant N protein & Recombinant $\mathrm{N}$ protein \\
\hline Target & $\begin{array}{l}\text { TOSV IgM/IgG SFSV, SFNV, } \\
\text { SFCV IgM/IgG }\end{array}$ & TOSV IgM/IgG & $\begin{array}{l}\text { TOSV IgM/IgG Hantavirus } \\
\text { IgM/IgG }\end{array}$ \\
\hline Sample & Serum/plasma & Serum/plasma & Serum/plasma \\
\hline Turnaround time & $\sim 1 \mathrm{~h} 15 \mathrm{~min}$ & $\sim 1 \mathrm{~h} 50 \mathrm{~min}$ & $\sim 4 \mathrm{~h} 45 \mathrm{~min}$ \\
\hline Hands-on time & $\sim 15 \min$ & $\sim 15 \min$ & $\sim 45 \mathrm{~min}$ \\
\hline Interpretation & Fluorescent microscopy & Optic density measurement & Visual \\
\hline \multirow[t]{2}{*}{ Sensitivity $^{\mathrm{a}}$} & Not available & $100 \%(\mathrm{IgM})$ & $100 \%(\operatorname{IgM})$ \\
\hline & & $95.0 \%$ (IgG) & $100 \%$ (IgG) \\
\hline \multirow[t]{2}{*}{ Specificity $^{\mathrm{a}}$} & Not available & $100 \%$ (IgM) & $99 \%(\operatorname{IgM})$ \\
\hline & & $96.5 \%(\operatorname{IgG})$ & $99 \%(\operatorname{IgG})$ \\
\hline
\end{tabular}

${ }^{\mathrm{a}}$ As provided by the manufacturer.

IIFT, indirect immunofluorescence test; ELISA, enzyme-linked immunosorbent assay; IB, immunoblot; TOSV, Toscana virus; SFSV, Sicilian virus; SFNV, sandfly fever Naples virus; SFCV, sandfly fever Cyprus virus.

comparison of antibody titers were performed by SPSS package version 17.0 (SPSS Inc.).

\section{Results}

\section{Detection of SFV/TOSV IgG and IgM seroreactivity by commercial assays}

The distribution of positive/equivocal/tested results and antibody titers for SFV serotypes according to the IIFT substrate are given in Table 2. Overall rate of equivocal/positive results from TOSV IIFT and ELISA were 18/53 (total: 71/120; $59.2 \%$ ) and 10/8 (total:18/120; 15\%), respectively. Positive/ strong positive results from TOSV IgG IB were $9 / 43$ (total:52/ 120; 43.3\%) (Table 2). No reactivity for Hantavirus serotypes was observed in any of the IB reactive samples.

For SFV IgM, a total of eight samples $(8 / 120,6.7 \%)$ were reactive (positive or equivocal) in the IIFT (Table 2 ). The distribution of SFV serotypes identified was SFNV in 3, SFSV in 2 , TOSV in 1, and SFSV+SFCV in 1 sample. IgM IIFT titers observed are given in Table 2 . All samples were negative with the TOSV IgM ELISA, and one SFNV IgM IIFT reactive sample was positive in TOSV IgG ELISA. IB assay for TOSV IgM was not performed.

\section{VNT results}

VNTs for SFSV, SFNV, and TOSV were performed for 99 samples, excluding 21 due to insufficient amounts. A total of 31 samples $(31 / 99 ; 31.3 \%)$ were reactive in the VNTs that comprise 15 TOSV (15.2\%), 11 SFSV (11.1\%), and 3 SFNV $(3.03 \%)$ (Table 3$)$. Two samples were observed to possess neutralizing antibodies for more than one SFV serotype and displayed reactivity for SFSV and SFNV where the titer of SFSV (1/320) exceeded that of SFNV (1/40 and 1/80) (Table 3). None of the IgM-positive samples were reactive in VNTs, and for the sample with SFSV+SFCV IgM positivity, VNT was not performed. The presence of neutralizing antibodies for TOSV could be demonstrated in 15 of the 89 samples $(16.9 \%)$ reactive in any of the commercial assays and in 15 of 60 samples $(25 \%)$ reactive with TOSV IIFT. In samples with IIFT reactivity other than TOSV, $41.9 \%(13 / 31)$ and $9.7 \%$
(3/31) were positive in VNTs for SFSV and SFNV, respectively (Table 3 ).

\section{Evaluation of TOSV commercial assays}

A total of 31 samples $(31 / 120 ; 25.8 \%)$ were observed as negative for TOSV IgG and 89 samples (89/120; 74.2\%) were reactive in at least one of the commercial assays (positive and equivocal results interpreted as reactive). Thirteen samples $(13 / 89 ; 14.6 \%)$ were reactive in all assays. Reactivity in IIFT+IB and IIFT+ELISA only were noted in $25(28.1 \%)$ and 2 (2.2\%) samples, respectively. For equivocal samples in IIFT, 12 out of 18 were observed as negative in ELISA or IB. In 49 samples $(49 / 89 ; 55.1 \%)$, reactivity was observed via a single assay (31 in IIFT, 14 in IB, and 4 for ELISA). No significant correlation among IIFT titers, ELISA optical densities, and/or VNT titers could be demonstrated (data not shown).

Agreement among commercial assays, assessed via kappa coefficient, was observed as slight to fair, and percentage agreement values were calculated as 50\%-63\% (average: $57.8 \%$ ) (Table 4 ). When results from all commercial assays $(\mathrm{IIFT}+\mathrm{ELISA}+\mathrm{IB})$ were evaluated via Fleiss kappa, the overall agreement was noted as slight (K: 0.116). The agreement between VNT and the commercial assays was slight to fair, with percentage agreement values between $53 \%$ and $82 \%$ (average: $62.6 \%$ ) (Table 4). Fleiss kappa for VNT and the commercial assays also displayed slight agreement (K: 0.151).

\section{Discussion}

TOSV is one of the major viral pathogens involved in aseptic meningoencephalitis occurring during summer in Mediterranean countries and poses a threat to the residents as well as the travelers (Eitrem et al. 1991b, Charel et al. 2005). Due to the frequent co-circulation of various SFV serotypes that cross-react in serological assays, the detection of immune responses to TOSV can be challenging (Magurano and Nicoletti 1999, Dionisio et al. 2003). Although a number of commercial assays have been developed for the serological detection of TOSV exposure, data on the performances of these assays have not been available. In this study, we evaluated the most widely used commercial assays for detecting 


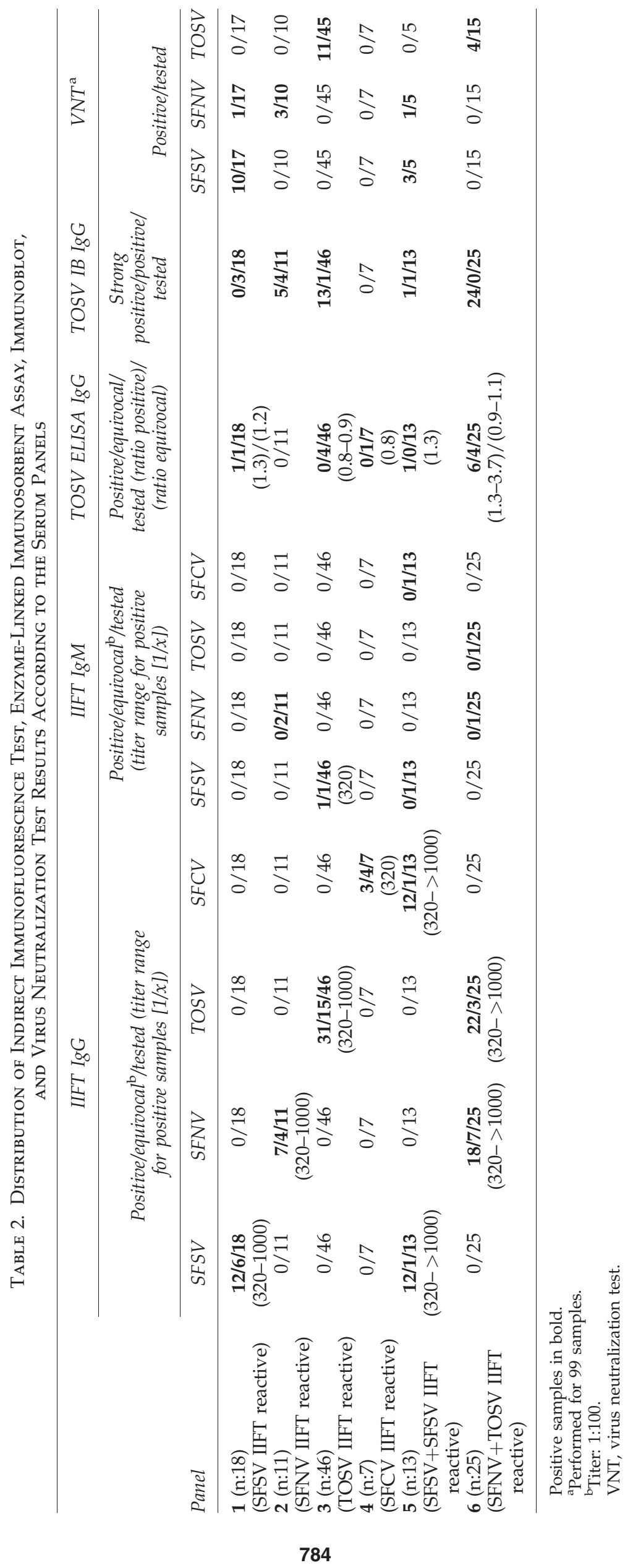


Table 3. Assay Results for the Virus Neutralization Test Reactive Samples

\begin{tabular}{|c|c|c|c|c|c|c|c|c|c|}
\hline \multirow[b]{2}{*}{ Sample no. } & \multicolumn{4}{|c|}{ IIFT (titer: $[1 / x]$ ) } & \multirow[b]{2}{*}{ ELISA (ratio) } & \multirow[b]{2}{*}{$I B$} & \multicolumn{3}{|c|}{ VNT (titer: $[1 / x]$ ) } \\
\hline & SFSV & SFNV & TOSV & SFCV & & & SFSV & SFNV & TOSV \\
\hline 1 & $\mathrm{n}$ & 320 & $\mathrm{n}$ & $\mathrm{n}$ & 0.459 & $1+$ & $\mathrm{n}$ & 160 & $\mathrm{n}$ \\
\hline 2 & $\mathrm{n}$ & 320 & $\mathrm{n}$ & $\mathrm{n}$ & 0.391 & $1+$ & $\mathrm{n}$ & 160 & $\mathrm{n}$ \\
\hline 3 & $\mathrm{n}$ & 320 & $\mathrm{n}$ & $\mathrm{n}$ & 0.496 & $2+$ & $\mathrm{n}$ & 80 & $\mathrm{n}$ \\
\hline 4 & 1000 & $\mathrm{n}$ & $\mathrm{n}$ & $\mathrm{n}$ & 1.305 & $\mathrm{n}$ & 320 & $\mathrm{n}$ & $\mathrm{n}$ \\
\hline 5 & 100 & $\mathrm{n}$ & $\mathrm{n}$ & $\mathrm{n}$ & 0.245 & $\mathrm{n}$ & 320 & $\mathrm{n}$ & $\mathrm{n}$ \\
\hline 6 & 320 & $\mathrm{n}$ & $\mathrm{n}$ & $\mathrm{n}$ & 0.481 & $1+$ & 320 & $\mathrm{n}$ & $\mathrm{n}$ \\
\hline 7 & 100 & $\mathrm{n}$ & $\mathrm{n}$ & $\mathrm{n}$ & 0.718 & $1+$ & 320 & 80 & $\mathrm{n}$ \\
\hline 8 & 320 & $\mathrm{n}$ & $\mathrm{n}$ & $\mathrm{n}$ & 0.162 & $1+$ & 320 & $\mathrm{n}$ & $\mathrm{n}$ \\
\hline 9 & 320 & $\mathrm{n}$ & $\mathrm{n}$ & $\mathrm{n}$ & 0.289 & $\mathrm{n}$ & 320 & $\mathrm{n}$ & $\mathrm{n}$ \\
\hline 10 & 100 & $\mathrm{n}$ & $\mathrm{n}$ & $\mathrm{n}$ & 0.093 & $\mathrm{n}$ & 320 & $\mathrm{n}$ & $\mathrm{n}$ \\
\hline 11 & 320 & $\mathrm{n}$ & $\mathrm{n}$ & $\mathrm{n}$ & 0.518 & $\mathrm{n}$ & 40 & $\mathrm{n}$ & $\mathrm{n}$ \\
\hline 12 & 320 & $\mathrm{n}$ & $\mathrm{n}$ & $\mathrm{n}$ & 0.185 & $\mathrm{n}$ & 80 & $\mathrm{n}$ & $\mathrm{n}$ \\
\hline 13 & 1000 & $\mathrm{n}$ & $\mathrm{n}$ & $\mathrm{n}$ & 0.082 & $\mathrm{n}$ & 320 & $\mathrm{n}$ & $\mathrm{n}$ \\
\hline 14 & $\mathrm{n}$ & $\mathrm{n}$ & 1000 & $\mathrm{n}$ & 0.450 & $2+$ & $\mathrm{n}$ & $\mathrm{n}$ & $40^{\mathrm{a}}$ \\
\hline 15 & $\mathrm{n}$ & $\mathrm{n}$ & 320 & $\mathrm{n}$ & 0.358 & $2+$ & $\mathrm{n}$ & $\mathrm{n}$ & $40^{\mathrm{a}}$ \\
\hline 16 & $\mathrm{n}$ & $\mathrm{n}$ & 1000 & $\mathrm{n}$ & 0.507 & $2+$ & $\mathrm{n}$ & $\mathrm{n}$ & $40^{\mathrm{a}}$ \\
\hline 17 & $\mathrm{n}$ & $\mathrm{n}$ & 320 & $\mathrm{n}$ & 0.879 & $2+$ & $\mathrm{n}$ & $\mathrm{n}$ & $40^{\mathrm{a}}$ \\
\hline 18 & $\mathrm{n}$ & $\mathrm{n}$ & 1000 & $\mathrm{n}$ & 0.873 & $2+$ & $\mathrm{n}$ & $\mathrm{n}$ & 40 \\
\hline 19 & $\mathrm{n}$ & $\mathrm{n}$ & 1000 & $\mathrm{n}$ & 0.684 & $1+$ & $\mathrm{n}$ & $\mathrm{n}$ & 40 \\
\hline 20 & $\mathrm{n}$ & $\mathrm{n}$ & 320 & $\mathrm{n}$ & 0.724 & $2+$ & $\mathrm{n}$ & $\mathrm{n}$ & 40 \\
\hline 21 & $\mathrm{n}$ & $\mathrm{n}$ & 320 & $\mathrm{n}$ & 0.366 & $2+$ & $\mathrm{n}$ & $\mathrm{n}$ & 40 \\
\hline 22 & $\mathrm{n}$ & $\mathrm{n}$ & 1000 & $\mathrm{n}$ & 0.784 & $3+$ & $\mathrm{n}$ & $\mathrm{n}$ & 40 \\
\hline 23 & $\mathrm{n}$ & $\mathrm{n}$ & 1000 & $\mathrm{n}$ & 0.633 & $2+$ & $\mathrm{n}$ & $\mathrm{n}$ & 40 \\
\hline 24 & $\mathrm{n}$ & $\mathrm{n}$ & 320 & $\mathrm{n}$ & 0.157 & $\mathrm{n}$ & $\mathrm{n}$ & $\mathrm{n}$ & 40 \\
\hline 25 & $\mathrm{n}$ & $>1000$ & $>1000$ & $\mathrm{n}$ & 3.661 & $3+$ & $\mathrm{n}$ & $\mathrm{n}$ & 40 \\
\hline 26 & $\mathrm{n}$ & $>1000$ & 1000 & $\mathrm{n}$ & 2.951 & $3+$ & $\mathrm{n}$ & $\mathrm{n}$ & 40 \\
\hline 27 & $\mathrm{n}$ & 320 & 1000 & $\mathrm{n}$ & 2.317 & $3+$ & $\mathrm{n}$ & $\mathrm{n}$ & 40 \\
\hline 28 & $\mathrm{n}$ & 100 & 320 & $\mathrm{n}$ & 0.898 & $3+$ & $\mathrm{n}$ & $\mathrm{n}$ & 40 \\
\hline 29 & $>1000$ & $\mathrm{n}$ & $\mathrm{n}$ & $>1000$ & 0.670 & $\mathrm{n}$ & 320 & $\mathrm{n}$ & $\mathrm{n}$ \\
\hline 30 & $>1000$ & $\mathrm{n}$ & $\mathrm{n}$ & $>1000$ & 0.430 & $2+$ & 320 & $\mathrm{n}$ & $\mathrm{n}$ \\
\hline 31 & 1000 & $\mathrm{n}$ & $\mathrm{n}$ & 1000 & 0.270 & $1+$ & 320 & 40 & $\mathrm{n}$ \\
\hline
\end{tabular}

$n$, Negative.

Shown in bold are ELISA equivalent/positive results.

${ }^{a}$ No further titration.

TOSV immunoglobulins. In addition, a significant number of the samples $(99 / 120 ; 82.5 \%)$ were investigated for the presence of neutralizing antibodies to SFSV, SFNV, and TOSV as well. Since IgM for TOSV and other SFVs were only detected in a small number of samples in the study group via IIFT/ ELISA (n:8) and VNTs were observed to be negative, we did not perform the IB assay for IgM reactive samples or compared IgM results. This constitutes the main limitation of this study. Nevertheless, viral RNA detection has been established as a powerful tool and an effective alternative for identifying acute TOSV infections (Charel et al. 2005). The impact of viral RNA detection in TOSV-associated central nervous system infections in the study region has also been documented recently, where TOSV IgM was identified via IIFT in only 4 (25\%) of the 16 TOSV RNA positive sera (Ergünay et al. 2010).

For TOSV IgG, the commercial assays evaluated in this study were observed to display slight to fair agreement with an average percentage of $57.8 \%$. This relatively low agreement observed is presumably due to the viral antigens employed, as well as differences in assay configurations, as displayed previously (Calisher et al. 1999, Cusi et al. 2001, Di Bonito et al. 2002). While virus-infected cells are the source of the antigens in the IIFT, recombinant $\mathrm{N}$ protein of TOSV is used in the ELISA and IB, although in different matrices. The TOSV IIFT

Table 4. Agreement Among Commercial Assays and Virus Neutralization Test For Toscana Virus IgG Detection

\begin{tabular}{lcccr}
\hline & IIFT (Kappa $/ \%$ agreement) & ELISA (Kappa/\% agreement) & IB (Kappa/\% agreement) & VNT (Kappa/\% agreement) \\
\hline IIFT & - & $0.111 / 50$ & $0.235 / 60.8$ & $0.179 / 53.3$ \\
ELISA & $0.111 / 50$ & - & $0.175 / 62.5$ & $0.248 / 81.7$ \\
IB & $0.235 / 60.8$ & $0.175 / 62.5$ & - & $0.278 / 67.5$ \\
\hline
\end{tabular}

${ }^{a}$ Interpreted $<0$ as no agreement and $0-0.20$ as slight, $0.21-0.40$ as fair, $0.41-0.60$ as moderate, $0.61-0.80$ as substantial, and $0.81-1$ as almost perfect agreement. 
titers were significantly higher in VNT reactive than nonreactive samples, with TOSV antibody titers equal to or higher than $1 / 320$ in all VNT reactive samples. Similar findings were also noted for the majority of the SFSV/SFNV reactive samples as well. Thus, the IIFT can be used for screening purposes, but equivocal evaluations might require retesting and/ or confirmation. A major advantage of the IIFT is the detection of the antibody responses against four different SFV serotypes, enabling identification of probable cross-reactions. Nevertheless, IIFT is a labor-intensive method that requires the individual examination of all samples in the fluorescent microscope, and the interpretation can vary according to the microscopist.

In TOSV ELISA, equivocal and positive results were equally distributed in VNT-reactive samples and in samples reactive via TOSV IIFT or IB (data not shown). Thus, equivocal results from this assay strongly imply reactivity. The TOSV IB assay displayed reactivity in all SFNV VNT-positive samples and in the majority of the SFNV and SFNV+TOSV IIFT reactive samples, which suggests cross-reactions with SFNV. However, this assay provided negative results in the majority of the IIFT or ELISA equivocal samples and positive results in the majority of the TOSV VNT-positive samples. Thus, IB can be employed as a confirmatory assay for TOSV exposure for samples reactive in TOSV IIFT or ELISA. The ELISA format enables rapid testing and interpretation in a high number of samples and can be easily automated. It has been previously reported that for TOSV, the performance of whole virus and recombinant nucleocapsid ELISAs correlate well (Valassina et al. 1998). However, the relatively lower sensitivity observed for the ELISA, as compared to IIFT and $\mathrm{VNT}$, suggests that the seroprevalence rates might have been underestimated in the previous studies where this assay was employed for surveillance (Echevarria et al. 2003, Valassina et al. 2003, Terrosi et al. 2009). The IB assay requires longer hands-on time than ELISA or IIFT but provides data about Hantavirus exposure (serotypes Hantaan, Puumala, Dobrava, Seoul) in the same strip as well.

An interesting finding is that VNT, which is generally regarded as the gold-standard assay for specificity, has provided confirmation only in a portion of the samples reactive for TOSV immunoglobulins via any assay and observed to be relatively insensitive. Moreover, the agreement of VNT and the commercial assays was interpreted as slight. It has been known that phleboviral neutralization is a complex phenomenon involving a number antigenic epitopes and even the majority of the exposed persons develop antibodies against linear epitopes of $\mathrm{N}$ protein, the immune response against viral glycoproteins, which affects virus neutralization in vitro, have been shown to display considerable variation (Besselaar and Blackburn 1991, Di Bonito et al. 2002). Patients with TOSV-induced neurological disease have also been observed to develop a differential antibody response against denatured viral proteins (Di Bonito et al. 2002). The presence of TOSV glycoprotein-precititating antibodies were associated with higher neutralizing antibody titers in acute and convelescent sera (Magurano and Nicoletti 1999). In addition, neutralizing epitopes may not necessarily be detected in diagnostic assays and may depend on tertiary structures that may be lost during assay development (Magurano and Nicoletti 1999, Di Bonito et al. 2002). These variations may account for the slight agreement observed among commercial diagnostic assays and VNT. A similar phenomenon has also been described for mosquito-borne flaviviruses West Nile virus and Yellow fever virus, where correlation of ratio/titers of ELISA or IIFT to VNT has observed to be low (Niedrig et al. 1999, Niedrig et al. 2007). Nevertheless, VNTs are still essential for seroepidemiological studies for confirming the presence of neutralizing antibodies. It also remains to be verified if surface glycoprotein variants of TOSV also co-circulate in endemic areas and are involved in human immune response with probable consequences in diagnostic serology. Nevertheless, it is likely that TOSV diagnostic assays will significantly benefit from inclusion of viral glycoprotein antigens in addition to nucleocapsid.

In conclusion, commercially available diagnostic immunoassays displayed slight-to-fair agreement as determined for IgG in sera from asymptomatic blood donors from an endemic region. The results could only be confirmed via virus neutralization in a portion of the samples, and overall agreement between the commercial assays and VNT is slight. The IIFT could be used for screening purposes, and for confirmation of TOSV exposure, commercial assays such as IB can be performed in addition to VNT.

\section{Acknowledgments}

This work was partially funded by Turkish Red Crescent Society and Hacettepe University Research Fund (Project NO: $08 \mathrm{O} 101$ 001) and by BMBF grant 01KI0710 "Research on Zoonotic Infectious Diseases' program; Emerging ArthropodBorne-Viral Infections in Germany: Pathogenesis, Diagnostics and Surveillance."

\section{Disclosure Statement}

Nadine Litzba received funding from the Euroimmun Medizinische Labordiagnostika AG, Germany. All authors declare that no further competing financial interests exist.

\section{References}

Besselaar, TG, Blackburn, NK. Topological mapping of antigenic sites on the Rift Valley fever virus envelope glycoproteins using monoclonal antibodies. Arch Virol 1991; 121:111-124.

Calisher, CH, Beaty, BJ, Chandler, LJ. Arboviruses. In: Lenette, EH, Smith, TF, eds. Laboratory Diagnosis of Viral Infections, 3rd edition. Revised and Expanded. New York: Marcel Dekker, 1999:305-332.

Charel, RN, Gallian, P, Navarro-Mari, JM, Nicoletti, L, et al. Emergence of Toscana virus in Europe. Emerg Infect Dis 2005; 11:1657-1663.

Ciufolini, MG, Fiorentini, C, Di Bonito, P, Mochi, S, et al. Detection of Toscana virus-specific immunoglobulins $\mathrm{G}$ and $\mathrm{M}$ by an enzyme-linked immunosorbent assay based on recombinant viral nucleoprotein. J Clin Microbiol 1999; 37:2010-2012.

Clarke, DH, Cassals, J. Techniques for hemagglutination and hemagglutination-inhibition with arthropod-borne viruses. Am J Trop Med Hyg 1958; 7:561-573.

Cusi, MG, Valensin, PE, Donati, M, Valassina, M. Neutralization of Toscana virus is partially mediated by antibodies to the nucleocapsid protein. J Med Virol 2001; 63:72-75.

Davies, FG, Jacobsen, P, Sylla, D. Laboratory manual on Rift Valley fever: isolation and identification technics. In: Report of FAO/WHO group on emergency preparedness for Rift Valley Fever control in West Africa (WHO-VPH/88.77). 1988:1-134. 
Di Bonito, P, Bosco, S, Mochi, S, Accardi, L, et al. Human antibody response to Toscana virus glycoproteins expressed by recombinant baculovirus. J Med Virol 2002; 68:615-619.

Dionisio, D, Esperti, F, Vivarelli, A, Valassina, M. Epidemiological, clinical and laboratory aspects of Sandfly Fever. Curr Opin Infect Dis 2003; 16:383-388.

Echevarria, JM, de Orf, F, Guisasola, ME, Sanchez-Seco, MP, et al. Acute meningitis due to Toscana virus infection among patients from both the Spanish Mediterranean region and the region of Madrid. J Clin Virol 2003; 26:79-84.

Eitrem, R, Niklasson, B, Weiland, O. Sandfly virus among Swedish tourists. J Infect Dis 1991b; 23:451-457.

Eitrem, R, Stylianou, M, Niklasson, B. High prevalence rates of antibody to three sandfly fever viruses (Sicilian, Naples, and Toscana) among Cypriots. Epidemiol Infect 1991a; 107:685-691.

Ergünay, K, Saygan, MB, Aydoğan, S, Lo, MM, et al. Sandfly Fever virus activity in Central/Northern Anatolia, Turkey: first report of Toscana virus infections. Clin Microbiol Infect 2010 [Epub ahead of print]; DOI: 10.1111/j.1469-0691.2010. 03346.x.

Hemmersbach-Miller, M, Parola, P, Charrel, RN, Durand, JP, et al. Sandfly fever due to Toscana virus: an emerging infection in southern France. Eur J Intern Med 2004; 15:316-317.

Ilstrup, DM. Statistical methods in microbiology. Clin Microbiol Rev 1990; 3:219-226.

Landis, JR, Koch, GG. The measurement of observer agreement for categorical data. Biometrics 1977; 33:159-174.

Magurano, F, Nicoletti, L. Humoral response in Toscana virus acute neurologic disease investigated by viral-protein-specific immunoassays. Clin Diagn Lab Immunol 1999; 6:55-60.

Mertz, GJ. Bunyaviridae: Bunyaviruses, Phleboviruses, Nairoviruses and Hantaviruses. In: Richman, DD, Whitley, RJ, Hayden, FG, eds. Clinical Virology. New York: ChurchillLivingstone, 1997:943-971.

Nicoletti, L, Ciufolini, MG, Verani, P. Sandfly fever viruses in Italy. Arch Virol 1996; 11:41-47.

Niedrig, M, Lademann, M, Emmerich, P, Lafrenz, M. Assessment of IgG antibodies directed against Yellow Fever virus after vaccination with 17D by different assays: neutralization test, haemagglutination inhibition test, immunofluorescence assay and ELISA. Trop Med Int Health 1999; 4:867-871.
Niedrig, M, Sonnenberg, K, Steinhagen, K, Paweska, JT. Comparison of ELISA and immunoassays for measurement of IgG and IgM antibody to West Nile virus in human sera against virus neutralisation. J Virol Methods 2007; 139:103-105.

Sanbonmatsu-Gamez, S, Perez-Ruiz, M, Collao, X, Sanchez-Seco, MP, et al. Toscana virus in Spain. Emerg Infect Dis 2005; 11:1701-1707.

Schwarz, TF, Jager, G, Gilch, S, Pauli, C. Serosurvey and laboratory diagnosis of imported sandfly virus, serotype Toscana, infection in Germany. Epidemiol Infect 1995; 114:501-510.

Schwarz, TF, Gilch, S, Pauli, C, Jager, G. Immunoblot detection of antibodies to Toscana virus. J Med Virol 1996; 49:83-86.

Schwarz, TF, Gilch, S, Schatzl, HM. A recombinant Toscana virus nucleoprotein in a diagnostic immunoblot system. Res Virol 1998; 149:413-418.

Soldateschi, D, dal Maso, GM, Valassina, M, Santini, L, et al. Laboratory diagnosis of Toscana virus infection by enzyme immunoassay with recombinant viral nucleoprotein. J Clin Microbiol 1999; 37:649-652.

Terrosi, C, Olivieri, R, Bianco, C, Cellesi, C, et al. Age-dependent seroprevalence of Toscana virus in central Italy and correlation with the clinical profile. Clin Vaccine Immunol 2009; 16:1251-1252.

Valassina, M, Soldateschi, D, dal Maso, GM, Santini, L, et al. Diagnostic potential of Toscana virus $\mathrm{N}$ protein expressed in Echerichia coli. J Clin Microbiol 1998; 36:3170-3172.

Valassina, M, Valentini, M, Pugliese, A, Valensin, PE, et al. Serological survey of Toscana virus infections in a high-risk population in Italy. Clin Diagn Lab Immunol 2003; 10:483-484.

Address correspondence to: Koray Ergünay Virology Unit

Department of Medical Microbiology Faculty of Medicine Hacettepe University Morphology

Building $3^{\text {rd }}$ Floor 06100 Ankara Turkey

E-mail: ekoray@hacettepe.edu.tr 
